\title{
Repetitive Transcranial Magnetic Stimulation For The Treatment of Chronic Tinnitus: A Preliminary Study of The Influence of Traumatic Brain Injury on Treatment Response
}

\author{
Garret A. Horton ${ }^{1 *}$ \\ Omar Ibrahim ${ }^{1}$ \\ Marcus Jansen ${ }^{2}$ \\ Jessica Trier $^{2}$ \\ Roumen $\mathrm{Milev}^{3}$ \\ Jason A. Beyea ${ }^{1,4}$
}

\begin{abstract}
This study aims to test whether the efficacy of repetitive Transcranial Magnetic Stimulation (rTMS) differs between patients who developed tinnitus following a traumatic brain injury (TBI), and those without a history of TBI. This was a parallel pilot, open-label, non-randomized, clinical trial to compare the efficacy of low frequency rTMS on tinnitus symptoms in patients with and without a TBI history. Patients with moderate to severe tinnitus symptoms based on the Tinnitus Handicap Inventory (THI) and the Tinnitus Functional Index (TFI) were enrolled in the study. Validated questionnaires (THI and TFI) were used to quantify the severity of tinnitus symptoms and hearing impairment (Hearing Handicap Index - HHI) before and after ten sessions of rTMS of the left primary auditory cortex. Hearing threshold levels as well as speech reception and speech discrimination thresholds were also compared. The number of patients who experienced a reduction in their subjective tinnitus symptoms was greater and sustained longer in patients without a history of TBI. The same was seen with subjective symptoms of hearing impairment. In conclusion, our preliminary results suggest tinnitus patients without a history of TBI respond better to low frequency rTMS than those with a history of TBI, suggesting that treatments could be more effective if tailored to tinnitus etiology.
\end{abstract}

Keywords: Tinnitus, Tinnitus, TBI, rTMS

'Department of Otolaryngology, Queen's University Kingston Ontario, Canada.

'Department of Physical Medicine and Rehabilitation, Queen's University Kingston, Ontario, Canada

'Departments of Psychiatry and Psychology, Queen's University, Kingston, Ontario, Canada

${ }^{4}$ Department of Otolaryngology, Otology, Neurology, and Cranial Base Surgery, Assistant Professor and ICES Adjunct Scientist, Queen's University School of Medicine, Kingston Health Sciences Centre, Ontario Canada

Department of Otolaryngology, Otology, Neurotology, and Cranial Base Surgery, Assistant Professor and ICES Adjunct Scientist, Queen's University School of Medicine, Kingston Health Sciences Centre, Ontario Canada, E-mail: jason.beyea@queensu.ca Phone: +16135443400

Paper submitted on February 10, 2021; and Accepted on March 01, 2021 


\section{INTRODUCTION}

Tinnitus is the perception of sound in the absence of auditory stimuli. This condition affects approximately $10 \%$ of adults and $53 \%$ of people who have experienced a Traumatic Brain Injury (TBI) ${ }^{1,2}$. There is currently no cure for tinnitus; current treatments focus on symptom management. The pathophysiology of tinnitus is not yet fully understood, hindering the development of effective treatments3. Tinnitus can result from traumatic insults to the auditory system, such as exposure to loud noises, head and neck trauma, or from ototoxic medications; however, in many cases the cause of tinnitus is unknown. It is hypothesized that tinnitus is ultimately the result of neuroplastic changes that promote spontaneous neuronal activity within the auditory system ${ }^{3}$. In support of this, positron emission tomography studies examining the brains of patients with tinnitus have shown significantly increased metabolic activity in the primary auditory cortex. Reducing aberrant neural activity within the primary auditory cortex could theoretically serve as a therapeutic target for tinnitus treatment ${ }^{4}$.

Repetitive transcranial magnetic stimulation (rTMS) is a method of non-invasive neural modulation which can reduce neural activity in discrete brain regions. rTMS works by directing an oscillating magnetic field at a defined region of the brain, effectively entraining the targeted neurons to the frequency of the oscillating magnetic field. Thus, applying low frequency rTMS to auditory centers in the brain has the potential to improve tinnitus symptoms by reducing spontaneous neural activity through inhibitory entrainment. Previous studies have attempted to treat tinnitus using low frequency rTMS directed at auditory centers of the brain, however the results of these studies vary ${ }^{5-7}$. In a meta-analysis of 15 studies evaluating the efficacy of rTMS as a tinnitus treatment, 7 of the 12 studies using low frequency rTMS found symptoms to be significantly reduced at 1 and 6 months post treatment7. However, the other 5 low frequency trials found rTMS to be no more effective than the sham intervention ${ }^{7}$. In addition, a recent multicenter trial in Germany found that rTMS therapy was not superior to sham treatment 8 .

A potential explanation for the variable efficacy of rTMS in treating tinnitus may be the heterogeneity of the population analyzed to date. The etiology of tinnitus varies and patients with tinnitus of different etiologies may respond differently to treatment. With this in mind, little research has been performed on subpopulations of tinnitus patients. Specifically, despite the high rate of tinnitus in patients with TBI, research on the effectiveness of rTMS in this tinnitus sub-population is lacking. To our knowledge, only one case study has tested rTMS as a treatment for TBI-associated tinnitus and marked symptom improvement was reported9. We hypothesized that stratifying patients by disease etiology may unmask an effect of rTMS in certain patient populations. To that end, we designed two pilot parallel clinical trials, one consisting of patients with TBI-associated tinnitus and another with patients with idiopathic tinnitus, to test whether the efficacy of rTMS differs between these subpopulations.

\section{MATERIALS AND METHODS}

\section{Institutional Review Board}

This study was approved by the Queen's University Health Sciences \& Affiliated Teaching Hospitals Research Ethics Board (HSREB), project \#6017436.

\section{Participants}

A total of 24 patients suffering from chronic tinnitus were recruited from the Neurotology Clinic at Hotel Dieu Hospital and the Acquired Brain Injury Clinic at Providence Care Hospital. Patients were recruited directly from their clinic appointments in Kingston, Ontario, Canada. Study details were explained to potential study participants and a consent form outlining the potential risks and benefits associated with the study was provided for participants to sign'. Enrolment in the study was determined by symptom severity according to patients' scores on the Tinnitus Handicap Inventory (THI) and Tinnitus Functional Index (TFI). The THI and TFI are validated questionnaires designed to assess the severity of tinnitus symptoms ${ }^{10,11}$. Both questionnaires were utilized to allow for comparison with prior studies as the TFI has become the industry standard, but many prior studies have utilized the THI. Patients with moderate or severe tinnitus symptoms (THI score $\geq 38$ and TFI score $\geq 32$ ) were eligible for study enrolment. Patients were excluded from the study if they were chronic alcohol users ( $>14$ drinks per week for men, $>7$ drinks per week for women), had a seizure disorder (or have had an early or late post-traumatic seizure), had a family history of epilepsy, had metallic implants in their head or neck, were pregnant, or taking benzodiazepines, opioids, prescription stimulants, insulin, immunosuppressant's or prednisone. Patients under 18 years of age were excluded.

\section{Study Design}

The design included parallel pilot open-label, nonrandomized clinical trials to determine the efficacy of a low frequency rTMS protocol in patients with TBI-associated chronic tinnitus and in patients where no cause was identified.

\section{Intervention}

The rTMS protocol was designed to replicate previous studies that found rTMS to significantly reduce tinnitus symptoms ${ }^{6}$. The protocol consisted of 10 daily sessions, each consisting of 2000 pulses delivered to the left primary auditory cortex on consecutive business days. These pulses were delivered at a frequency of $1 \mathrm{~Hz}$ and at an intensity of $110 \%$ of the motor threshold, i.e. the magnetic pulse strength necessary to trigger contraction of the abductor pollicis brevis muscle of the thumb. All 
rTMS treatments were conducted by an experienced rTMS-trained nurse, who routinely performs clinical treatments of patients with rTMS for other medical conditions. Coil placement was based on the standard procedure of coil positioning developed by Langguth et al. ${ }^{12}$. During the first session, the subject donned a fitted cap, which was reused at each subsequent session. The midpoint $(\mathrm{Cz})$ between the nasion and inion was then measured and marked on the cap. The distance (D1) between the zygomatic arches (indent anterior to the tragus) was measured in a line through $\mathrm{Cz}$. A mark (A) was then made on the cap $10 \%$ of the distance from the left zygomatic arch to the right, plus $2.5 \mathrm{~cm}$. $A$ final mark (B) was made $1.5 \mathrm{~cm}$ posterior to mark $A$ and perpendicular to line D1. Mark B was above the left primary auditory cortex, and the rTMS coil was placed above this point for the duration of the rTMS protocol. At each subsequent treatment, the cap from the first session was donned and arranged in the original position to target the primary auditory cortex. The left primary auditory cortex was selected as studies have found increased neuronal activity primarily in the left primary auditory cortex of patients with tinnitus; however, right sided increases have been reported ${ }^{4-13}$.

\section{Outcomes}

At the time of recruitment, the tinnitus symptoms were evaluated using the THI and TFI. In addition, symptoms of hearing impairment were assessed using the Hearing Handicap Inventory for Adults (HHIA) as well as by a routine clinical audiogram performed by an audiologist. Upon completion of the last session of rTMS, tinnitus symptoms and hearing were reassessed using the same questionnaires. Questionnaires were performed again at 2 weeks and 2 months post intervention. An audiogram was performed 2 months after rTMS. Audiogram values and subjects' HHIA questionnaire scores were used to assess changes in hearing as a secondary outcome since tinnitus is often associated with hearing impairment ${ }^{14}$.

\section{Statistical Analysis}

The effect of rTMS on participant's tinnitus symptoms was assessed by comparing baseline THI, TFI, and HHIA questionnaire scores collected prior to the intervention and scores immediately following the last session of rTMS.
Baseline scores were also compared with scores at 2 weeks and 2 months after the intervention. An intention to treat, last observation carried forward analysis was used to analyze the questionnaire data of these participants. A one-way ANOVA was utilized for this analysis. Hearing outcomes were assessed using audiogram data collected at baseline and 2 months post treatment completion. Patients lacking follow-up audiogram data were excluded from analysis. For all comparisons, differences were considered significant if the null hypothesis was rejected at $p<0.05$. This data was analyzed using a one-way ANOVA and paired samples T-test.

\section{RESULTS}

\section{Tinnitus Symptoms: THI and TFI Questionnaires}

A total of 12 participants completed the intervention protocol; 5 with and 7 without a history of TBI. Two of the five patients with a history of TBI that completed the rTMS protocol were lost to follow-up; one after completion of the post treatment assessment, and the other after the 2-week assessment. The mean THI and TFI scores in patients with and without a history of TBI are shown in Figures 1a and $1 \mathrm{~b}$. When analyzed as a single cohort, $41.7 \%$ of patients with tinnitus showed a 10 point or greater improvement in THI questionnaire scores 2 months after rTMS treatment (Table 1). However, when analyzed based on TBI status $57.1 \%$ of tinnitus patients without a history of TBI and $0 \%$ with, had a 10 point or greater improvement in their THI scores after 2 months. Similarly, when analyzed as a single cohort $8.3 \%$ of tinnitus patients showed a 10 point or greater improvement in TFI questionnaire scores 2 months after rTMS treatment. However, when analyzed based on TBI status $14.3 \%$ of tinnitus patients without a history of TBI and $0 \%$ with, had a 10 point or greater improvement in their TFI scores after 2 months. Despite these promising findings, a one-way ANOVA of THI questionnaire scores revealed that there was no significant interaction between time relative to rTMS treatment and questionnaire score $(p=0.360)$, or between time relative to rTMS, TBI status, and questionnaire score $(p=0.665)$. Sphericity was violated in both cases therefore a Greenhouse-Geisser correction was used. There was no significant interaction between questionnaire scores and TBI status $(p=0.442)$. Analysis of TFI questionnaire scores showed no significant interaction between time
A)

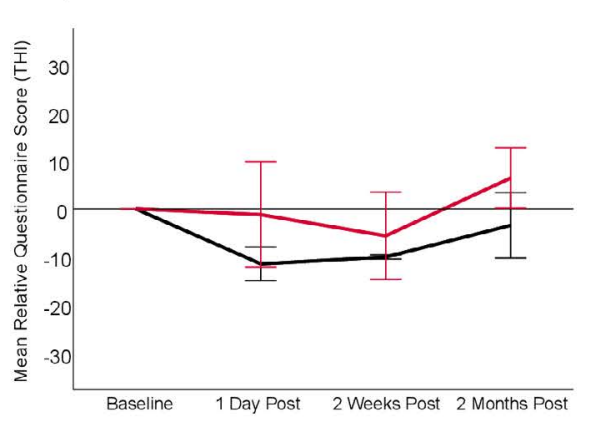

B)

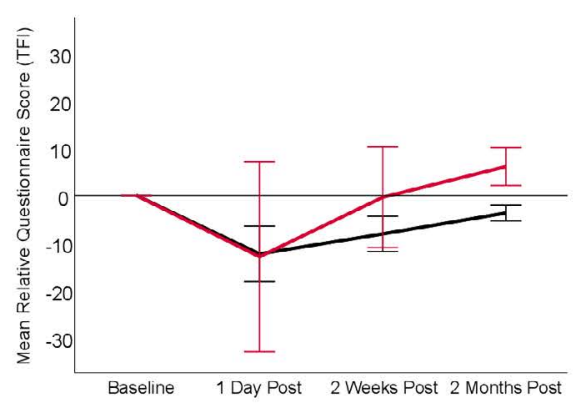

C)

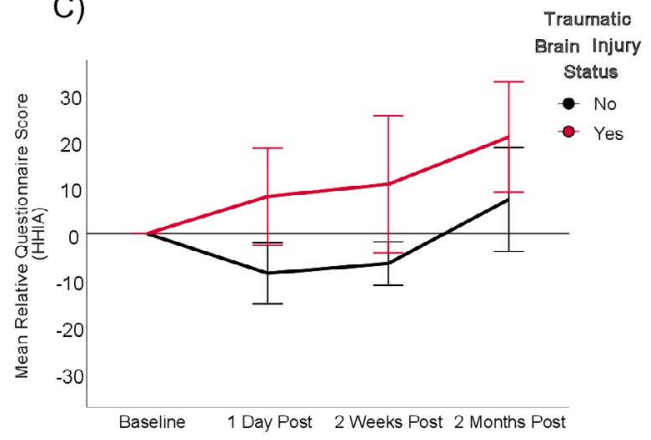

Figure 1: THI and TFI scores in patients with and without a history of TBI. 
Table 1: Percentage of patients within each sub-population who exhibited reduced questionnaire scores is shown. Point reduction indicates the percentage of patients whose questionnaire scores were reduced by $>X$ number of points at each time point. The colour of each cell is a visual marker of the percentage value within it. A darker shade of green indicates a greater percentage of the subject population.

\begin{tabular}{|c|c|c|c|c|c|c|c|c|c|c|}
\hline & \multirow[b]{2}{*}{$\begin{array}{c}\text { Point } \\
\text { Reduction }\end{array}$} & \multicolumn{3}{|c|}{ All Participants } & \multicolumn{3}{|c|}{ Non-TBI } & \multicolumn{3}{|c|}{ TBI } \\
\hline & & $\begin{array}{l}1 \text { Day } \\
\text { Post }\end{array}$ & $\begin{array}{l}2 \text { Weeks } \\
\text { Post }\end{array}$ & $\begin{array}{l}2 \text { Months } \\
\text { Post }\end{array}$ & $\begin{array}{l}\text { 1 Day } \\
\text { Post }\end{array}$ & $\begin{array}{l}2 \text { Weeks } \\
\text { Post }\end{array}$ & $\begin{array}{l}2 \text { Months } \\
\text { Post }\end{array}$ & $\begin{array}{l}1 \text { Day } \\
\text { Post }\end{array}$ & $\begin{array}{l}2 \text { Weeks } \\
\text { Post }\end{array}$ & $\begin{array}{l}2 \text { Months } \\
\text { Post }\end{array}$ \\
\hline THI & $>0$ & 75 & 75 & 50 & 100 & 100 & 71.4 & 40 & 40 & 20 \\
\hline$>10$ & 50 & 58.3 & 41.7 & 57.1 & 85.7 & 57.1 & 40 & 20 & 0 & \\
\hline$>20$ & 16.7 & 8.3 & 0 & 14.3 & 0 & 0 & 20 & 20 & 0 & \\
\hline$>30$ & 16.7 & 8.3 & 0 & 14.3 & 0 & 0 & 20 & 20 & 0 & \\
\hline$>40$ & 0 & 8.3 & 0 & 0 & 0 & 0 & 20 & 0 & 0 & \\
\hline TFI & $>0$ & 66.7 & 58.3 & 58.3 & 85.7 & 71.4 & 85.7 & 40 & 40 & 20 \\
\hline$>10$ & 41.7 & 33.3 & 8.3 & 42.9 & 42.9 & 14.3 & 40 & 20 & 0 & \\
\hline$>20$ & 25 & 16.7 & 0 & 28.6 & 14.3 & 0 & 20 & 20 & 0 & \\
\hline$>30$ & 16.7 & 8.3 & 0 & 14.3 & 0 & 0 & 20 & 20 & 0 & \\
\hline$>40$ & 16.7 & 0 & 0 & 14.3 & 0 & 0 & 20 & 0 & 0 & \\
\hline HHIA & $>0$ & 50 & 50 & 41.7 & 71.4 & 71.4 & 71.4 & 20 & 20 & 0 \\
\hline$>10$ & 25 & 25 & 8.3 & 28.6 & 28.6 & 14.3 & 20 & 20 & 0 & \\
\hline$>20$ & 16.7 & 16.7 & 0 & 14.3 & 14.3 & 0 & 20 & 20 & 0 & \\
\hline$>30$ & 16.7 & 16.7 & 0 & 14.3 & 14.3 & 0 & 20 & 20 & 0 & \\
\hline$>40$ & 8.3 & 0 & 0 & 14.3 & 0 & 0 & 0 & 0 & 0 & \\
\hline
\end{tabular}

relative to rTMS treatment and questionnaire score $(p=0.180)$, or between time relative to rTMS treatment, TBI status, and questionnaire score $(p=0.644)$. Sphericity was violated in this case as well, therefore a GreenhouseGeisser correction was used. There was no significant interaction between questionnaire scores and TBI status $(p=0.997)$.

\section{Symptoms of Hearing Impairment: HHIA Questionnaire}

The mean HHIA scores in patients with and without a history of TBI are shown in figure 1c. When analyzed as a single cohort, $8.3 \%$ of patients with tinnitus showed a 10 point or greater improvement in their HHIA questionnaire score 2 months after rTMS treatment (Table 1). However, when analyzed based on TBI status $14.3 \%$ of tinnitus patients without a history of TBI and $0 \%$ with, had a 10 point or greater improvement in their HHIA scores after 2 months. Despite these findings, a one-way ANOVA showed no significant interaction between time relative to rTMS treatment and HHIA questionnaire score $(p=0.442)$ or between time relative to rTMS treatment, TBI status, and questionnaire score $(p=0.428)$. There was no significant interaction between HHIA questionnaire scores and TBI status $(p=0.635)$.

\section{Symptoms of Hearing Impairment: HHIA Questionnaire}

Two TBI patients were excluded from this analysis as they were lost to follow-up and lacked post-treatment audiogram data. Of the remaining 10 participants, 2 lacked meaningful hearing in their left ear (1 with and 1 without a history of TBI). Because of this the left ear data has a ' $n$ ' of 8 ( 2 with and 6 without a history of TBI) and $\mathrm{n}=10$ for the right ear data (7 with and 3 without a history of TBI). Hearing threshold levels (HTLs) were measured between 250 and $8000 \mathrm{~Hz}$ prior to and 2 months after rTMS treatment. As participants' pre-treatment HTLs varied, HTL values measured at 2 months were normalized to preintervention levels to facilitate inter-subject comparison and derive more meaningful mean and range values as presented in Table 2 and Figure 2. When analyzed as a single cohort, a one-way repeated measures ANOVA analyzing the normalized HTLs in both ears found no significant interaction between time relative to rTMS treatment and hearing response threshold $(p=0.284)$. In addition, there was no significant interaction between hearing response threshold, TBI status, and time relative to rTMS treatment $(p=0.564)$. There was no significant interaction between hearing response threshold and ear tested $(p=0.807)$. There was a significant interaction between hearing response threshold and frequency $(p<0.001)$. There was no significant interaction between hearing response threshold, frequency, and TBI status $(p=0.276)$. There was no significant interaction between hearing response threshold and TBI status $(p=0.243)$. Seeing a marked decrease in the graphed high frequency HTLs in the left ear of patients with tinnitus without a history of TBI (Figure 2b), a post-hoc paired samples t-test was performed to directly compare hearing threshold levels at each measured frequency, prior to and 2 months after rTMS. When all patients with tinnitus were analyzed together, a significant reduction in HTL was identified in the left ear at 3000 and $4000 \mathrm{~Hz}(p=0.049$ and 0.011 respectively). There was no significant change in HTL in either ear at any other frequency when analyzed as a single group. Interestingly, when participants were 
Table 2: Mean hearing threshold levels (HTLs) in all subjects $(A)$ and those with $(C)$ and without $(B)$ a history of TBI at 2 months post rTMS normalized to levels prior to treatment (baseline). Values are separated by ear and frequency tested. the range of normalized HTL values post rTMS are also shown. p-values are derived from a paired samples T-test of corresponding raw, non-normalized HTLs at baseline and 2 months post treatment. Data corresponds to figure 2. Hearing threshold levels (HTLs) pre and post-rTMS treatment.

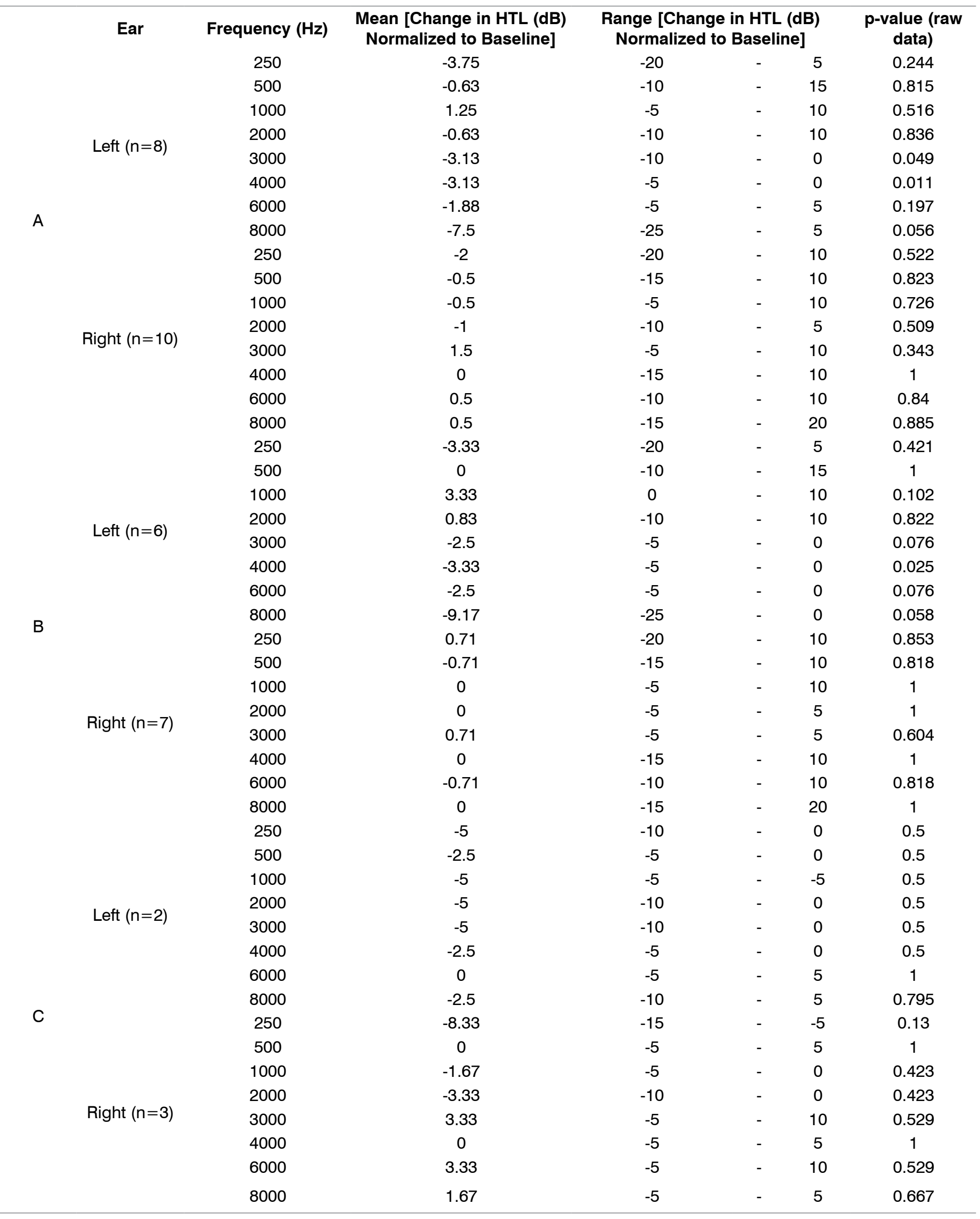



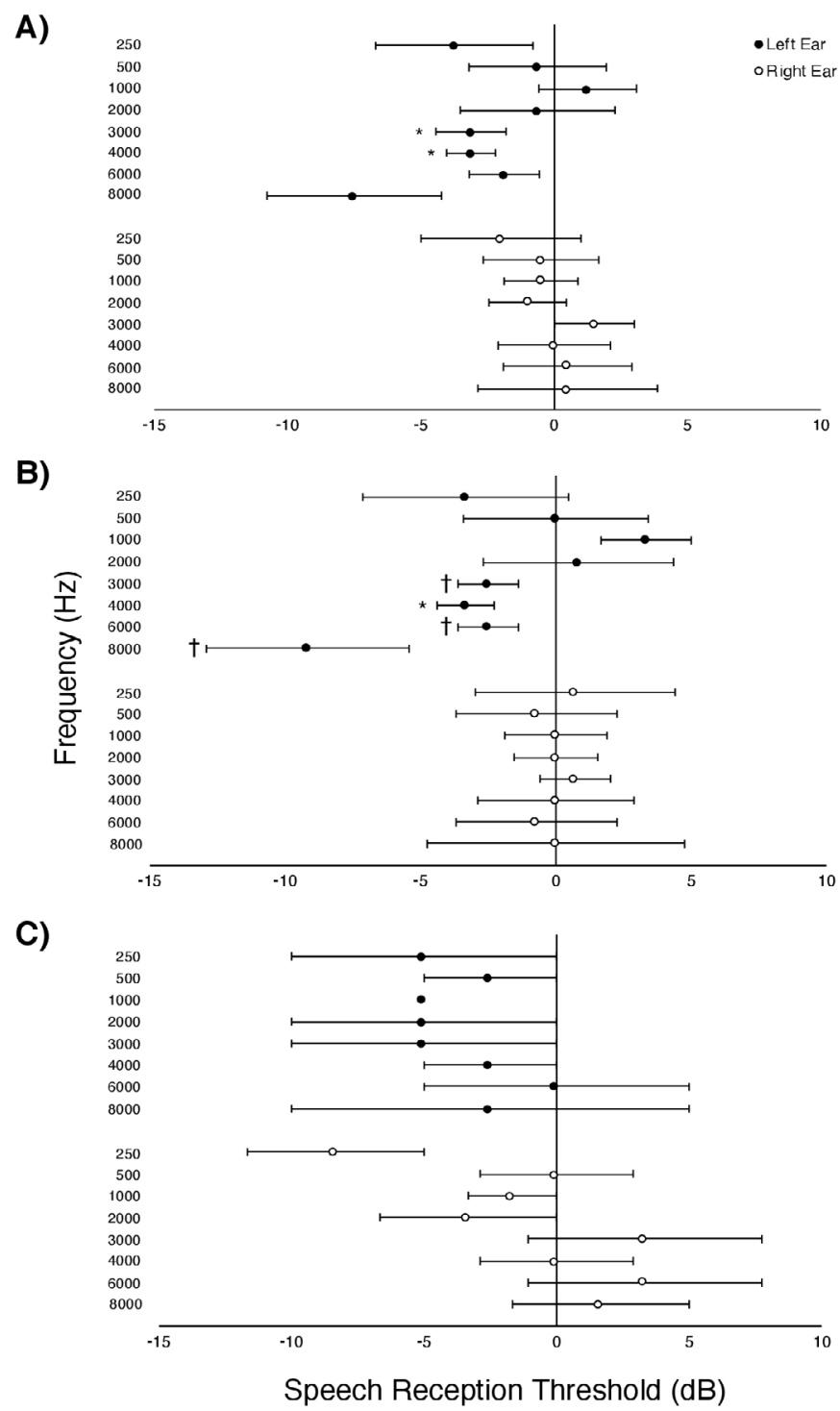

Figure 2: ANOVA analyzing the normalized HTLs in both ears found no significant interaction between time relative to rTMS treatment.

subdivided based on history of TBI, patients without a history of TBI showed a significant decrease in HTL measured in the left ear at $4000 \mathrm{~Hz}(p=0.025)$ and trended towards a significant decrease at 3000,6000 , and $8000 \mathrm{~Hz}$ in the left ear $(p<0.1)$. A significant change in HTL was not observed in the right ear at any frequency in either subgroup. A significant change in HTL was not observed at any frequency in either ear in patients with a history of TBI. This data is shown in Table 2 and Figure 2. Each participant's speech reception threshold (SRT) was also assessed at baseline and at 2 months post rTMS. The SRT is a measure of the minimum sound intensity in decibels at which an individual can correctly identify $50 \%$ of spoken words. When the data from both ears was analyzed together, there was no significant interaction between treatment and speech reception threshold $(p=0.374)$. There was no significant interaction between speech reception threshold and TBI status $(p=0.368)$. There was also no significant interaction between speech reception threshold, $\mathrm{TBI}$ status, and time relative to rTMS $(p=0.818)$. There was no significant interaction between speech reception threshold and the ear tested $(p=0.666)$. As a measure of hearing impairment, speech discrimination at baseline and at 2 months post rTMS was compared using a surrogate value composed of the percentage of correct words divided by the volume level in decibels at which the score was achieved (ie. $100 \%$ correct at $50 \mathrm{dBHL}$ returns a value of 2 ). Analysis of both ears together showed no significant interaction between time relative to rTMS and speech discrimination $(p=0.594)$. There was not a significant interaction between speech reception threshold and TBI status $(p=0.445)$. No significant interaction was observed between speech discrimination, TBI status, and time relative to $\operatorname{rTMS}(p=0.372)$. There was not a significant interaction between speech discrimination and ear tested $(p=0.907)$. Importantly, of all subjects enrolled in the study $33 \%$ of those with a history of TBI chose to withdraw from the study due to discomfort or a perceived worsening of their symptoms. In contrast, $14 \%$ of patients without a history of TBI withdrew from the study. 


\section{DISCUSSION}

Tinnitus is a common condition that disproportionately affects those who have experienced a TBI1,2. Prior studies have had mixed findings with regards to the effectiveness of rTMS as a treatment for tinnitus ${ }^{5-7}$. We hypothesized those patients with differing tinnitus etiologies may respond differently to rTMS and thus assessed the efficacy of rTMS in patients with and without a history of TBI. In this study, the number of patients who experienced a reduction in their subjective tinnitus symptoms was greater and lasted longer in the non-TBI group than the TBI group. The same was seen with subjective symptoms of hearing impairment and suggests that patients with tinnitus without a history of TBI respond better to rTMS than those with a history of TBI. For this reason, investigating the efficacy of tinnitus treatments in specific patient subpopulations could reveal improved treatment efficacy when compared to the effect in all tinnitus patients. This opens new avenues of investigation for tinnitus treatments based on etiology. Improved questionnaire scores were seen at the longest time-point post treatment (2 months) in the majority of patients with tinnitus who did not have a history of TBI. This was not seen in patients with a history of TBI. Interestingly, the sustained symptom improvement seen in non-TBI participants aligns with previous studies that have shown sustained improvement in tinnitus symptoms months after $1 \mathrm{~Hz}$ rTMS directed at the left auditory cortex 12-15. Conversely, the lack of sustained improvement seen in TBI participants aligns with prior studies that did not observe a sustained symptom improvement despite a significant initial improvement ${ }^{16-18}$. In addition, this study showed HTLs to preferentially improve in the left ear of tinnitus patients without a history of TBI. This finding supports our initial hypothesis that patients with tinnitus of differing etiologies will respond differently to treatment. However, we did not anticipate quantitative hearing improvement in the ear ipsilateral to the primary auditory cortex targeted with low frequency rTMS. To our knowledge, this is the first time that this has been shown. Lastly, this study showed improved tinnitus symptoms and significantly improved left ear HTLs in participants without TBI at $4000 \mathrm{~Hz}$ and trended towards a significant improvement at 3000,6000 , and $8000 \mathrm{~Hz}$. This aligns with the hypothesis that reducing aberrant neural activity in brain regions associated with high frequency auditory signals can improve tinnitus symptoms and associated hearing impairment. However, further research is needed to correlate improved HTLs at these frequencies with reduced brain activity following rTMS in the associated regions of the primary auditory cortex. Finally, this study was hampered by small sample sizes as a result of low enrollment and high drop-out rates amongst participant with TBI. Nearly one third of all tinnitus patients enrolled with a history of TBI withdrew from the study or did not return for follow-up. This was more than double the number of participants without TBI that chose to drop-out of the study. Reasons for choosing to discontinue participation in the study were not formally collected, however many of the participants with TBI reported discomfort during rTMS and a perceived worsening of their tinnitus symptoms as the cause. Participants with TBI may also experience memory impairment, which may affect follow through with respect to appointments. Although rTMS is non-invasive, it can be associated with noise exposure of up to $145 \mathrm{~dB}$ 19. Ear plugs are used to combat this, but noise sensitivity is commonly experienced by patients with TBI. This should be taken into consideration when contemplating rTMS treatment for tinnitus patients with a history of TBI. Our preliminary results suggest that tinnitus of differing etiologies may respond differently to low frequency rTMS. Patients without a history of TBI may respond better to low frequency rTMS than patients with TBI. This could be attributed in part to the noise sensitivity often associated with traumatic brain injuries. These preliminary findings support the need to stratify patients with tinnitus based on etiology to more accurately assess the efficacy of potential tinnitus treatment modalities.

\section{CONCLUSION}

This study will serve as a platform from which to launch larger multicenter trials analyzing the effectiveness of rTMS in TBI-related tinnitus patients and those with idiopathic tinnitus. Such a trial has the potential to fill a gap that persists in the literature and support further investigations into the efficacy of rTMS in other tinnitus patient subpopulations.

\section{CONFLICT OF INTEREST}

The authors declare no potential conflict of interest

\section{REFERENCES}

1. Davis A, El Refaie A. Tinnitus Handbook in: Tyler RS edition. Ear and Hearing Singular. 2001;1:1-169.

2. Jury MA, Flynn MC. Auditory and vestibular sequelae to traumatic brain injury: a pilot study. NZ Med J. 2001;114:286-8.

3. Baguley D, McFerran D, Hall D. Tinnitus. Lancet. 2013;382:1600-7.

4. Arnold W, Bartenstein P, Oestreicher E, Romer W, Schwaiger $M$. Focal metabolic activation in the predominant left auditory cortex in patients suffering from tinnitus: a PET study with 18F deoxyglucose. ORL. 1996;58:195-9.

5. Theodoroff SM, Folmer RL. Repetitive transcranial magnetic stimulation as a treatment for chronic tinnitus: a critical review. Otol. Neurotol. 2003;34:199-208.

6. Langguth B, de Ridder D, Dornhoffer JL, Eichhammer P, Folmer RL, Frank E, et al. Controversy: does repetitive transcranial magnetic stimulation/transcranial direct current stimulation show efficacy in treating tinnitus patients? Brain Stimul. 2003;1:192-205.

7. Soleimani R, Jalali MM, Hasandokht $T$. Therapeutic impact of repetitive transcranial magnetic stimulation (rTMS) on tinnitus: a systematic review and meta-analysis. Eur. Arch. Oto-Rhino-Laryngology. 2003;273:1663-75. 
8. Landgrebe M, Hajak G, Wolf S, Padberg F, Klupp $P$, Fallgatter $\mathrm{AJ}$, et al. $1-\mathrm{Hz}$ rTMS in the treatment of tinnitus: a sham-controlled, randomized multicenter trial. Brain Stimul Basic Transl Clin Res. 2017;10:1112-20.

9. Kreuzer PM, Landgrebe M, Frank E, Langguth B. Repetitive transcranial magnetic stimulation for the treatment of chronic tinnitus after traumatic brain injury: a case study $\mathrm{J}$ Head Trauma Rehabil. 2013;28:386-9.

10. Newman C, Jacobson G, Spitzer J. Development of the tinnitus handicap inventory. Arch. Otolaryngol. 2003;122:143-8.

11. Meikle MB, Henry JA, Griest SE, Stewart BJ, Abrams HB, McArdle R. The tinnitus functional index: development of a new clinical measure for chronic, intrusive tinnitus Ear Hear. 2012;33:153-176.

12. Langguth $B$, Zowe M, Landgrebe $M$, Sand $P$, Kleinjung $T$, Binder, $\mathrm{H}$, et al. Transcranial magnetic stimulation for the treatment of tinnitus: a new coil positioning method and first results. Brain Topogr. 2006;18:241-7.

13. Smits M, Kovacs S, De Ridder D, Peeters RR, Van Hecke $P$, Sunaert, $S$. Lateralization of Functional Magnetic Resonance Imaging (fMRI) activation in the auditory pathway of patients with lateralized tinnitus. Neuroradiology. 2009;49:669-79.
14. Ochi K, Ohashi T, Kenmochi M. Hearing impairment and tinnitus pitch in patients with unilateral tinnitus: comparison of sudden hearing loss and chronic tinnitus. Laryngoscope. 2013;113:427-31.

15. Kleinjung T, Steffens T, Sand P, Murthum T, Hajak G, Strutz $J$, et al. Which tinnitus patients benefit from transcranial magnetic stimulation? Otolaryngol. 2007;137:589-95.

16. Plewnia C, Reimold M, Najib A, Reischl G, Plontke SK, Gerloff $C$, et al. Moderate therapeutic efficacy of positron emission tomography-navigated repetitive transcranial magnetic stimulation for chronic tinnitus: a randomised, controlled pilot study. J Neurol Neurosurg Psychiatry. 2011;78:152-6.

17. Rossi S, De Capua A, Ulivelli M, Bartalini S, Falzarano V, Filippone $\mathrm{G}$, et al. Effects of repetitive transcranial magnetic stimulation on chronic tinnitus: a randomised, crossover, double blind, placebo controlled study. J. Neurol. Neurosurg. 2007;78:857-63.

18. Langguth $B$, Kleinjung T, Frank E, Landgrebe M, Sand $P$, Dvorakova $\mathrm{J}$, et al. High-frequency priming stimulation does not enhance the effect of low-frequency rTMS in the treatment of tinnitus. Exp brain Res. 2007;184:587-91.

19. Counter SA, Borg E. Analysis of the coil generated impulse noise in extracranial magnetic stimulation. Electroencephalogr Clin Neurophysiol Potentials Sect. 1992;85:280-88. 